\title{
(อ) OPEN ACCESS \\ Three-year follow-up of accelerated transepithelial corneal cross-linking for progressive paediatric keratoconus
}

\author{
Mi Tian, ${ }^{1,2}$ Weijun Jian, ${ }^{1,2}$ Xiaoyu Zhang, ${ }^{1,2}$ Ling Sun, ${ }^{1,2}$ Xingtao Zhou (1) 1,2
}

\begin{abstract}
${ }^{1}$ Department of Ophthalmology, Eye \& ENT Hospital, Fudan University, Shanghai, China ${ }^{2}$ NHC Key Laboratory of Myopia, Shanghai, China
\end{abstract}

\section{Correspondence to} Dr Xingtao Zhou, Fudan University, Shanghai 200433, China; doctzhouxingtao@163. com

MT and WJ contributed equally.

Received 18 September 2019 Revised 22 December 2019 Accepted 28 January 2020

\section{Check for updates}

(c) Author(s) (or their employer(s)) 2020. Re-use permitted under CC BY-NC No commercial re-use. See rights and permissions. Published by BMJ.

To cite: Tian $\mathrm{M}$, Jian $\mathrm{W}$

Zhang $\mathrm{X}$, et al. Br J

Ophthalmol

2020;104:1608-1612.
ABSTRACT

Purpose To investigate the long-term safety and efficacy of accelerated transepithelial corneal cross-linking (ATE-CXL) in children with progressive keratoconus.

Methods Fifty-three eyes of 41 paediatric patients (34 boys, 7 girls; mean age $14.81 \pm 1.96$ years) undergoing ATE-CXL were enrolled in the study. Corrected distance visual acuity (CDVA) and manifest refraction were assessed preoperatively and 36 months postoperatively. Corneal keratometry, corneal thickness and posterior elevation were measured using Pentacam preoperatively and 1, 6, 12 and 36 months postoperatively. Pachymetry and epithelial thicknesses were measured using optical coherence tomography preoperatively and 6,12 , and 36 months postoperatively.

Results Thirty-six months postoperatively, CDVA improved from $0.32 \pm 0.28$ to $0.26 \pm 0.25$ in logarithm of the minimum angle resolution $(p=0.025)$. Maximum keratometry was $58.73 \pm 9.70 \mathrm{D}$ preoperatively and 59.20 $\pm 10.24,58.28 \pm 9.33,57.88 \pm 9.99$ and $58.98 \pm 10.79$ D at $1,6,12$ and 36 months postoperatively throughout the 36-month follow-up period ( $p>0.05$ ). Similarly, corneal central thickness, which was $492.42 \pm 33.83 \mu \mathrm{m}$ postoperatively, also remained stable during the 36month follow-up ( $p>0.05$ ). Both posterior central elevation and posterior highest elevation were stable at 12 months after ATE-CXL ( $>>0.05)$, but increased at 36 months postprocedure $(p<0.05)$. Corneal pachymetry and epithelial thicknesses remained stable throughout the follow-up period ( $p>0.05$ ).

Conclusions ATE-CXL is a safe and effective treatment in paediatric progressive keratoconus patients, leading to stable keratometry and corneal thickness throughout the 36-month follow-up.

\section{INTRODUCTION}

Keratoconus is a bilateral and progressive corneal ectasia, which causes corneal thinning and steepening, leading to irregular astigmatism and a decrease in visual acuity. ${ }^{1}$ It usually occurs during puberty and progresses until the third or fourth decade of life. ${ }^{2}$ Paediatric keratoconus is more aggressive than the adult variant. Diagnosis of this disorder before adulthood is a risk factor for progression, increasing the probability of the need for a corneal transplant. ${ }^{3}$ Therefore, more attention should be paid to the diagnosis and treatment of paediatric keratoconus in early stages of the disease. ${ }^{4}$
Corneal cross-linking (CXL) can increase the biomechanical strength and stability of the cornea by an interaction of ultraviolet (UV) light and riboflavin, which is used to halt or decelerate the progression of keratoconus. ${ }^{5}$ Previous studies $^{6-10}$ have reported that conventional corneal collagen cross-linking (C-CXL; epitheliumoff procedure with $3 \mathrm{~mW} / \mathrm{cm}^{2}$ UVA radiation for $30 \mathrm{~min}$ ) is an effective and safe procedure to treat adult and paediatric keratoconus. Only a few studies ${ }^{11}{ }^{12}$ have investigated the use of accelerated transepithelial (ATE) CXL (ATECXL; epithelium-on procedure with $45 \mathrm{~mW} / \mathrm{cm}^{2}$ UVA radiation in the pulsed mode for $320 \mathrm{~s}$ ) for the treatment of paediatric keratoconus.

Here, we report for the first time the results of a 3-year follow-up of ATE-CXL in children with progressive keratoconus. This study investigated the long-term safety and efficacy of ATECXL for paediatric patients with progressive keratoconus.

\section{PATIENTS AND METHODS \\ Patient population}

Paediatric patients (10-17 years old) with progressive keratoconus who underwent ATE-CXL at the Eye and Ear Nose Throat Hospital of Fudan University in Shanghai, China were enrolled in this study. The diagnosis of keratoconus was based on the global consensus on keratoconus and ectatic diseases criteria. ${ }^{13}$ Written informed consent was obtained from the guardian of each patient before enrolment and after a detailed explanation of the procedure.

Inclusion criteria were diagnosis with progressive keratoconus with the thinnest corneal thickness (TCT) greater than or equal to $400 \mu \mathrm{m}$. Keratoconus progression was confirmed by an increase in maximum keratometry (Kmax) by 1.00 diopter (D) or more in 1 year, or an increase in astigmatic degree of $1.00 \mathrm{D}$ or more in 1 year. Exclusion criteria included advanced keratoconus with stromal scarring, a history of other ocular diseases, previous ocular surgeries or allergy to riboflavin. Patients wearing rigid gas permeable lenses or soft contact lenses discontinue use of these for at least 4 or 2 weeks, respectively.

Fifty-three eyes of 41 patients $(34$ males and 7 females) with a mean age of $14.81 \pm 1.96$ years were included in this study. The baseline characteristics of the study population are summarised in table 1 . 


\begin{tabular}{lcc}
\hline Table 1 & The baseline characteristics of the patients \\
\hline & Mean \pm SD & Range \\
\hline Age (years) & $14.81 \pm 1.96$ & 10 to 17 \\
Sphere (D) & $-5.35 \pm 4.30$ & -18.50 to 0.50 \\
Cylinder (D) & $-4.24 \pm 2.41$ & -0.50 to -8.75 \\
Spherical equivalent (D) & $-7.51 \pm 4.39$ & 0 to 20.88 \\
CDVA (logMAR) & $0.32 \pm 0.28$ & 0 to 1.30 \\
K1 (D) & $47.13 \pm 5.27$ & 41.3 to 64.4 \\
K2 (D) & $51.28 \pm 6.16$ & 42.6 to 68.0 \\
Kmax (D) & $58.73 \pm 9.70$ & 43.9 to 82.1 \\
CCT ( $\mu$ m) & $492.42 \pm 33.83$ & 427 to 578 \\
AT $(\mu \mathrm{m})$ & $483.89 \pm 37.76$ & 409 to 576 \\
TCT $(\mu \mathrm{m})$ & $474.91 \pm 36.45$ & 400 to 563 \\
\hline
\end{tabular}

AT, apex thickness; CCT, central corneal thickness; CDVA, corrected distance visual acuity; D, diopters; K1, steepest meridian keratometry; K1, flattest meridian keratometry; Kmax, maximum keratometry; logMAR, logarithm of the minimal angle of resolution; TCT, thinnest corneal thickness.

\section{Preoperative and postoperative measurements}

Preoperative and postoperative examinations included slitlamp biomicroscopy, manifest refraction, corrected distance visual acuity (CDVA) in logarithm of the minimum angle resolution $(\log M A R)$, corneal topography (Pentacam, Oculus, Wetzlar, Germany) and spectral-domain optical coherence tomography (OCT; RTVue-100; Optovue, Fremont, California, USA). The last follow-up was 3 years after ATE-CXL. All measurements were performed by the same experienced technician.

Steepest meridian keratometry (K1), flattest meridian keratometry (K2), Kmax, central corneal thickness (CCT), apex thickness (AT) and TCT were measured by the Pentacam system. For each eye, the best-fit sphere in the central $8.0 \mathrm{~mm}$ zone of the preoperative cornea was used as the reference surface. The Pentacam system automatically measured posterior central elevation (PCE) and posterior highest elevation (PHE) of the cornea. Posterior mean elevation (PME) was calculated as the mean of 27 points in the central $4.0 \mathrm{~mm}$ zone of the posterior corneal surface. Changes in elevation ( $\triangle \mathrm{PCE}, \triangle \mathrm{PHE}$ and $\triangle \mathrm{PME}$ ), which were calculated by subtracting preoperative from postoperative data, were considered the shift of the posterior corneal surface. The followed-up time points were $1,6,12$ and 36 months postoperatively.

Spectral-domain OCT was used to measure sectoral epithelium thickness and sectoral pachymetry thickness of the central cornea (the central $2 \mathrm{~mm}$ diameters), the paracentral cornea (the central annuli of diameters from 2 to $5 \mathrm{~mm}$ ) and the mid-peripheral cornea (the central annuli of diameters from 5 to $6 \mathrm{~mm}$ ) postoperatively and at 1,12 and 36 months following ATE-CXL.

\section{Surgical procedure}

All ATE-CXL procedures were performed by the same surgeon (XZho) following a previously described procedure. ${ }^{12}{ }^{14-16}$ The corneal epithelium was left intact. Corneal soaking of riboflavin was performed using Paracel $(0.25 \%$ riboflavin-5-phosphate, hydroxypropyl methylcellulose, sodium edetate, trometamol, benzalkonium chloride and $\mathrm{NaCl}$, Avedro, USA) for 4 min and VibeX Xtra (0.25\% riboflavin-5-phosphate and $\mathrm{NaCl}$, Avedro) for $6 \mathrm{~min}$. The UV treatment was administered using $365 \mathrm{~nm} \mathrm{UV-}$ A light (Avedro's KXL System, Avedro) for $5 \mathrm{~min}$ and $20 \mathrm{~s}$ with $45 \mathrm{~mW} / \mathrm{cm}^{2}$ irradiation in the pulsed mode ( $1 \mathrm{~s}$ on and $1 \mathrm{~s}$ off). The bandage contact lens was removed on postoperative days $1-5$, according to epithelialisation. Prescriptions were given for
Table 2 Preoperative and postoperative refraction and visual acuity (mean \pm SD)

\begin{tabular}{lccc}
\hline Parameter & Preoperation & 36 months postoperation & P value \\
\hline Sphere (D) & $-5.35 \pm 4.30$ & $-5.84 \pm 4.11$ & 0.050 \\
Cylinder (D) & $-4.24 \pm 2.41$ & $-4.32 \pm 2.71$ & 0.681 \\
Spherical equivalent (D) & $-7.51 \pm 4.39$ & $-8.01 \pm 4.14$ & 0.052 \\
CDVA (logMAR) & $0.32 \pm 0.28$ & $0.26 \pm 0.25$ & $0.025^{*}$
\end{tabular}

${ }^{*} A$ significant difference was detected.

CDVA, corrected distance visual acuity; $D$, diopters; logMAR, logarithm of the minimal angle of resolution.

levofloxacin eye-drops, to be applied four times per day for 1 week, artificial tears to be used four times per day for 1 month, and $0.1 \%$ fluorometholone to be used seven times daily initially, followed by gradual reduction over 3 weeks.

\section{Data and statistical analysis}

Statistical analysis was performed using SPSS V.20.0 (SPSS). Continuous parameters were described as mean \pm SD. Normality of the data was tested using the Kolmogorov-Smirnov test. Paired t-test, Wilcoxon rank-sum test and repeated measures analyses of variance with Bonferroni-adjusted post hoc comparisons were performed to evaluate the significance of differences between preoperative and postoperative data. Differences with $\mathrm{p}<0.05$ were considered statistically significant.

\section{RESULTS}

All surgical procedures were completed successfully, without any intraoperative or postoperative complications. All patients were observed using slitlamp biomicroscopy after bandage lens removal at postoperative days $1-5$. There were no cases of corneal haze or infection.

\section{Visual acuity and refraction}

CDVA (in logMAR units) and refraction data obtained at baseline and 3 years postoperatively are shown in table 2 . A significant improvement in CDVA was observed between the postoperative time point and 36-month follow-up $(0.32 \pm 0.28$ vs $0.26 \pm 0.25$; $\mathrm{p}=0.025)$. No significant changes were found in spherical equivalent refraction (SE), which was $-7.51 \pm 4.39 \mathrm{D}$ preoperatively and $-8.01 \pm 4.14 \mathrm{D}$ at 3 years post-ATE-CXL $(\mathrm{p}=0.052)$.

At 36 months postoperatively, CDVA improved in 18 eyes $(36 \%)$, remained stable in 26 eyes $(52 \%)$ and decreased in 6 eyes $(12 \%)$.

\section{Corneal keratometry}

Maximum keratometry was $58.73 \pm 9.70 \mathrm{D}$ preoperatively, and $59.20 \pm 10.24,58.28 \pm 9.33,57.88 \pm 9.99$ and $58.98 \pm 10.79 \mathrm{D}$ at 1, 6, 12 and 36 months after ATE-CXL, respectively. There was no significant difference in $\mathrm{K} 1, \mathrm{~K} 2$ and $\mathrm{Kmax}$ between preoperative and postoperative time points spanning the 36-month follow-up ( $\mathrm{p}>0.05$; figure $1 \mathrm{~A})$.

At 3 years postoperatively, Kmax decreased by $1.00 \mathrm{D}$ or more in 17 eyes $(34 \%)$, changed from $-1.00 \mathrm{D}$ to $1.00 \mathrm{D}$ in 23 eyes $(46 \%)$, and increased by $1.00 \mathrm{D}$ or more in 10 eyes (20\%).

\section{Corneal thickness and posterior elevation}

CCT, AT and TCT of preoperative and postoperative measurements are summarised in figure 1B. CCT values were $492.42 \pm 33.83 \mu \mathrm{m}$ postoperatively and $492.31 \pm 35.05$, $496.22 \pm 34.67,496.44 \pm 31.55$ and $488.16 \pm 31.61 \mu \mathrm{m}$ at 1,6 , 12 , and 36 months postoperatively. There was no statistically 

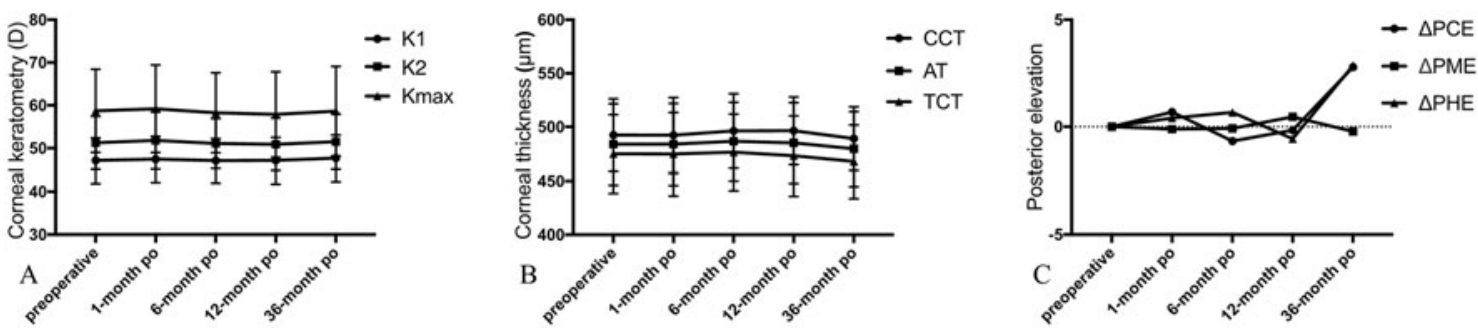

Figure 1 (A) The K1, K2 and Kmax values were stable during the 36-month follow-up ( $p>0.05$ ). There is no statistical significance between each time point. K1, steepest meridian keratometry; K1, flattest meridian keratometry; Kmax, maximum keratometry. (B) The CCT, AT and TCT values were stable during the 36-month follow-up ( $p>0.05$ ). There is no statistical significance between each time point. (C) The PCE and PME values showed nonsignificant changes in the first postoperative 12 months ( $p>0.05$ ), and significantly increased at 36 months after ATE-CXL ( $p<0.05)$. No significant changes were observed in PME during 36-month follow-up ( $p>0.05$ ). AT, apex thickness; ATE-CXL, accelerated transepithelial corneal cross-linking; CCT, central corneal thickness; PCE, posterior central elevation; PHE, posterior highest elevation; PME, posterior mean elevation; TCT, thinnest corneal thickness.

significant difference between any of these parameters during the 36-month follow-up period ( $\mathrm{p}>0.05)$.

Compared with baseline, PCE and PHE were not significantly different at the 1,6 and 12 months follow-up ( $p>0.05$ ), whereas at 36 months following ATE-CXL both of these parameters were significantly increased $(\mathrm{p}<0.05$; figure $1 \mathrm{C})$. No significant changes were observed in PME at any time point during followup $(\mathrm{p}>0.05)$.

\section{Epithelial and pachymetry thicknesses}

Sectoral epithelium thicknesses before and after ATE-CXL are listed in table 3. There was no significant change in sectoral epithelium thickness between preoperative and follow-up time points $(p>0.05)$, except for the paracentral superior sector, the thickness of which was significantly increased at 36 months postoperatively $(p=0.036)$. Minimum epithelial thicknesses were $42.10 \pm 7.18 \mu \mathrm{m}$ at baseline and $43.44 \pm 7.52 \mu \mathrm{m}$ at 36 months postoperatively $(\mathrm{p}=0.397)$.

Table 3 Sectoral epithelial thickness at each time point before and after ATE-CXL (mean \pm SD)

\begin{tabular}{lllll}
\hline \multirow{2}{*}{$\begin{array}{l}\text { Epithelial } \\
\text { thicknesses }(\mu \mathrm{m})\end{array}$} & Preoperation & 6 months & 12 months & 36 months \\
\hline Mininum & $42.10 \pm 7.18$ & $42.55 \pm 6.46$ & $41.65 \pm 8.50$ & $43.44 \pm 7.52$ \\
\hline Centre & $51.23 \pm 5.34$ & $51.98 \pm 5.60$ & $52.53 \pm 6.16$ & $51.73 \pm 5.84$ \\
\hline Paracentral-S & $56.27 \pm 5.39$ & $56.45 \pm 7.06$ & $57.50 \pm 7.25$ & $58.22 \pm 5.09$ \\
\hline Paracentral-SN & $57.85 \pm 5.93$ & $56.85 \pm 6.28$ & $58.50 \pm 6.41$ & $58.88 \pm 4.93$ \\
\hline Paracentral-N & $58.00 \pm 5.20$ & $57.45 \pm 5.37$ & $58.56 \pm 6.18$ & $57.76 \pm 4.81$ \\
\hline Paracentral-IN & $56.90 \pm 4.83$ & $54.98 \pm 9.94$ & $55.62 \pm 4.86$ & $56.20 \pm 6.33$ \\
\hline Paracentral-I & $53.88 \pm 6.69$ & $53.05 \pm 7.22$ & $51.68 \pm 6.88$ & $54.00 \pm 6.36$ \\
\hline Paracentral-IT & $50.31 \pm 5.47$ & $49.55 \pm 6.61$ & $48.29 \pm 6.37$ & $50.76 \pm 6.32$ \\
\hline Paracentral-T & $50.92 \pm 6.17$ & $51.90 \pm 6.21$ & $52.44 \pm 7.57$ & $52.17 \pm 6.73$ \\
\hline Paracentral-ST & $55.83 \pm 6.10$ & $57.15 \pm 7.67$ & $57.71 \pm 7.82$ & $57.05 \pm 7.17$ \\
\hline Mid-peripheral-S & $53.40 \pm 6.14$ & $53.88 \pm 6.37$ & $54.82 \pm 7.71$ & $56.29 \pm 5.69 *$ \\
\hline Mid-peripheral-SN & $56.10 \pm 5.13$ & $55.25 \pm 5.48$ & $56.12 \pm 5.90$ & $57.73 \pm 4.70$ \\
\hline Mid-peripheral-N & $59.46 \pm 4.90$ & $58.03 \pm 4.31$ & $58.32 \pm 4.92$ & $59.56 \pm 4.83$ \\
\hline Mid-peripheral-IN & $60.38 \pm 4.54$ & $59.80 \pm 5.28$ & $58.62 \pm 4.47$ & $60.22 \pm 5.85$ \\
\hline Mid-peripheral-I & $58.15 \pm 7.41$ & $57.25 \pm 8.21$ & $55.97 \pm 7.40$ & $59.66 \pm 6.24$ \\
\hline Mid-peripheral-IT & $55.27 \pm 7.09$ & $53.70 \pm 7.74$ & $52.62 \pm 7.84$ & $55.80 \pm 6.16$ \\
\hline Mid-peripheral-T & $55.38 \pm 6.51$ & $56.33 \pm 5.42$ & $56.24 \pm 6.38$ & $56.07 \pm 5.43$ \\
\hline Mid-peripheral-ST & $56.77 \pm 6.41$ & $57.60 \pm 5.52$ & $57.56 \pm 5.66$ & $58.34 \pm 5.29$ \\
\hline & & & & \\
\hline
\end{tabular}

${ }^{*}$ A significant difference was detected.

ATE-CXL, accelerated transepithelial corneal cross-linking; I, inferior; N, nasal; S, superior; T, temporal.
Sectoral pachymetry thicknesses before and after ATE-CXL are shown in table 4. Minimum pachymetry thicknesses were 461.23 $\pm 34.75 \mu \mathrm{m}$ at baseline and $457.95 \pm 39.90 \mu \mathrm{m}$ at 36 months postoperatively $(p=0.686)$. No significant changes were observed between sectoral pachymetry thicknesses at any time point during a follow-up ( $p>0.05)$.

\section{DISCUSSION}

Keratoconus has been shown to be more aggressive in the paediatric as compared with the adult population, ${ }^{17}$ with an increased risk of corneal opacities and a greater need for penetrating keratoplasty. ${ }^{3} 4$ The 1 -year progression of keratoconus in children occurred in $88 \%$ of the patients. ${ }^{18}$ Therefore, the treatment of keratoconus is particularly important in children. CXL is an effective treatment in stabilising keratoconus. ATE-CXL is a novel modification of this treatment that maintains the integrity of the corneal epithelium layer and has a higher UV irradiation intensity, allowing patients a faster recovery after a more comfortable treatment process. To the best of our knowledge, this is the

Table 4 Sectoral pachymetry thickness at each time point before and after ATE-CXL (mean \pm SD)

\begin{tabular}{lllll}
\hline \multirow{2}{*}{$\begin{array}{l}\text { Pachymetry } \\
\text { thicknesses }(\mu \mathrm{m})\end{array}$} & Preoperation & 6 months & 12 months & 36 months \\
\hline Mininum & $461.23 \pm 34.75$ & $461.70 \pm 37.88$ & $456.62 \pm 40.23$ & $457.95 \pm 39.90$ \\
\hline Centre & $487.85 \pm 30.88$ & $488.05 \pm 34.18$ & $485.74 \pm 33.74$ & $482.41 \pm 33.97$ \\
\hline Paracentral-S & $548.35 \pm 30.46$ & $548.48 \pm 32.55$ & $548.35 \pm 30.74$ & $547.17 \pm 31.24$ \\
\hline Paracentral-SN & $546.69 \pm 29.41$ & $544.93 \pm 29.20$ & $545.09 \pm 29.99$ & $544.78 \pm 29.28$ \\
\hline Paracentral-N & $532.25 \pm 26.95$ & $530.63 \pm 28.15$ & $524.94 \pm 28.11$ & $529.90 \pm 26.91$ \\
\hline Paracentral-IN & $516.81 \pm 27.72$ & $514.65 \pm 29.88$ & $504.56 \pm 36.19$ & $516.49 \pm 30.65$ \\
\hline Paracentral-I & $504.08 \pm 33.70$ & $501.83 \pm 34.74$ & $493.82 \pm 37.92$ & $510.27 \pm 32.66$ \\
\hline Paracentral-IT & $493.47 \pm 29.38$ & $491.03 \pm 35.78$ & $487.53 \pm 34.97$ & $495.29 \pm 32.66$ \\
\hline Paracentral-T & $503.77 \pm 29.92$ & $505.65 \pm 34.63$ & $505.94 \pm 32.33$ & $501.44 \pm 35.48$ \\
\hline Paracentral-ST & $534.35 \pm 28.96$ & $534.93 \pm 33.70$ & $534.88 \pm 30.18$ & $531.68 \pm 32.86$ \\
\hline Mid-peripheral-S & $579.69 \pm 35.52$ & $577.48 \pm 33.52$ & $681.63 \pm 35.75$ & $575.98 \pm 35.70$ \\
\hline Mid-peripheral-SN & $578.46 \pm 32.19$ & $574.55 \pm 30.40$ & $576.12 \pm 34.40$ & $573.41 \pm 32.75$ \\
\hline Mid-peripheral-N & $565.56 \pm 30.30$ & $562.75 \pm 29.36$ & $559.44 \pm 30.72$ & $563.98 \pm 28.97$ \\
\hline Mid-peripheral-IN & $543.21 \pm 73.95$ & $553.13 \pm 29.19$ & $547.26 \pm 33.22$ & $556.68 \pm 30.88$ \\
\hline Mid-peripheral-I & $549.58 \pm 34.36$ & $546.85 \pm 33.46$ & $541.15 \pm 34.38$ & $553.39 \pm 31.72$ \\
\hline Mid-peripheral-IT & $534.85 \pm 32.64$ & $533.58 \pm 35.33$ & $531.12 \pm 35.44$ & $540.49 \pm 31.66$ \\
\hline Mid-peripheral-T & $536.00 \pm 28.66$ & $539.28 \pm 33.11$ & $540.35 \pm 31.28$ & $537.37 \pm 31.91$ \\
\hline Mid-peripheral-ST & $564.56 \pm 31.42$ & $566.10 \pm 34.66$ & $568.50 \pm 32.72$ & $563.34 \pm 33.00$ \\
\hline ATE-CXL, & & & & \\
\hline
\end{tabular}

ATE-CXL, accelerated transepithelial corneal cross-linking; I, inferior; N, nasal; S, superior; T, temporal. 
first study to investigate the effects of ATE-CXL on progressive keratoconus in patients aged 10-17 years with a follow-up period of 36 months.

We observed an overall improvement in CDVA from 0.32 $\log$ MAR preoperatively to $0.26 \log$ MAR at 3 years postoperatively. Vinciguerra et $a l^{10}$ examined C-CXL as a treatment for paediatric keratoconus in 40 eyes of 40 patients and found a significant improvement of CDVA at 2 years following the treatment. Ozgurhan et al ${ }^{19}$ observed that CDVA improved significantly from $0.38 \pm 0.24 \log$ MAR preoperatively to $0.30 \pm 0.20$ logMAR at a 2-year follow-up of accelerated CXL for paediatric keratoconus in 44 eyes of 38 patients. Collectively, our results suggest that ATE-CXL is similarly as effective as C-CXL and accelerated CXL in the treatment of paediatric keratoconus in visual acuity assessment.

Our results showed that CDVA improved in 36\% of patient eyes, remained unchanged in 52\% eyes and decreased in 12\% eyes at 3 years postoperatively. According to the previous studies, Uçakhan et $a l^{9}$ examined C-CXL for keratoconus aged below 19 years and found at month 48, improvement by two or more lines in the mean CDVA was encountered in $77.5 \%$ of patient eyes and there was no loss of CDVA in any patient eye. However, not all studies demonstrated such significant improvements in visual outcomes. In Kumar Kodavoor et $a l^{20}$ study, there was improvement in the BCVA in 18 eyes (51.42\%), stabilisation in 12 eyes $(34.28 \%)$, and worsening of vision in 5 eyes (14.28\%) at 1-year follow-up of C-CXL for paediatric keratoconus.

We found that K1, K2, Kmax and corneal thickness tended to be stable throughout the follow-up period, and our patients had progressive keratoconus with an increase in Kmax of $1.00 \mathrm{D}$ or more in 1 year, suggesting that ATE-CXL is an effective treatment capable of halting the progression of keratoconus in paediatric patients. Our results were similar to results were found in adult keratoconus eyes reported by our group. ${ }^{14} 15$ Above-mentioned studies $^{10} 19$ had shown decrease of keratometry $(\mathrm{K})$ values at 2-year follow-up. However, not all studies have demonstrated significant decrease in $\mathrm{K}$ values. Wise $\mathrm{et}_{\mathrm{al}} \mathrm{l}^{7}$ reported in 39 eyes of 28 patients no significant change in mean $\mathrm{K}$ values at 1 -year follow-up after C-CXL treatment. Chatzis and Hafezi ${ }^{18}$ found the Kmax values were significantly reduced as early as 3 months after treatment and remained stable to 24 months after C-CXL in children and adolescents. However, at 36 months after C-CXL, the Kmax values showed a tendency for progression.

Kmax increased by $1.00 \mathrm{D}$ or more in $9.3 \%$ and $20.0 \%$ of patient eyes at 1 year and 3 years postoperatively. According to the previous studies of C-CXL for paediatric keratoconus, Kumar Kodavoor et $a l^{20}$ reported at 1 year postoperatively, $8.6 \%$ of patient eyes showed an increase in the Kmax, which were similar to our 1-year results. However, Ucakhan $e t a l^{9}$ found the mean Kmax increased by $1 \mathrm{D}$ only in $2.5 \%$ eyes at 2 years postoperatively. Above-mentioned studies did not have data for 36-month follow-up. In Schuerch et al ${ }^{6}$ study, 5 cases of 33 (15.15\%) eyes in a follow-up period of 37.5 months had an increase of $1 \mathrm{D}$ or more of Kmax. Chatzis and Hafezi ${ }^{18}$ investigated at 1, 2 and 3 years after C-CXL, Kmax readings were increased by $1 \mathrm{D}$ or more in $11 \%, 13 \%$ and $55 \%$ of eyes. The effect of CXL might not be strong enough to show arrest of keratoconus progression at longer follow-up.

In the current study, no complications were observed during the treatment, and no adverse reactions such as keratitis or corneal haze occurred after the treatment. Furthermore, CDVA improved during the 3-year follow-up, together providing evidence for the safety of ATE-CXL for treating paediatric keratoconus. In contrast, it has been reported that the use of C-CXL for the treatment of keratoconus can lead to corneal haze and during epithelial healing after C-CXL, the cornea is vulnerable to infection and melting. ${ }^{21} 22$ Therefore, ATE-CXL, not only maintaining the integrity of the corneal epithelium and consequently reducing the occurrence of complications, but also being more comfortable for the treated patients, is the superior method in this context.

In recent years, corneal posterior elevation was reliably used to evaluate the stability of the corneal structure $e^{23-25}$ in refractive surgery. We found that PCE and PHE slightly, though not statistically significantly, decreased during the 1-year follow-up. However, at the 3-year follow-up, both parameters were significantly increased compared with baseline, suggesting that ATECXL may have prevented or delayed the progress of corneal ectasia and maintained structural stability of the cornea at 1 year after ATE-CXL, but this beneficial effect was lost thereafter. Based on previous studies, ${ }^{26} 27$ in order to protect the corneal endothelium, the depth of cross-linking by riboflavin and UV radiation is generally in the anterior and middle stroma of the cornea. The occurrence of keratoconus usually starts from the posterior surface of the cornea. Therefore, during CXL treatment, the posterior surface of the cornea may not be adequately cross-linked. As the anterior stroma is more important in terms of biomechanical stability of the cornea, ${ }^{28}$ the cross-linking effect should also be strong enough to stop the progression of corneal ectasia.

Antonios et $a^{29}$ reported that OCT might better estimate the true CCT following CXL as compared with Scheimpflug tomography. With the use of OCT, we found that at 3 years postoperatively as compared with baseline, all sectors of pachymetry thickness as well as epithelial thickness showed no significant differences, except for the paracentral superior sector, which showed significantly increased thickness. In a previous study in which C-CXL was used for treating paediatric keratoconus, the authors reported that pupil centre pachymetry was significantly decreased at 3 months and recovered by 12 months postoperatively, remaining stable thereafter throughout the 2-year follow-up. ${ }^{10}$ Other studies ${ }^{29}{ }^{30}$ have demonstrated that corneal thickness decreases following epithelium-off crosslinking in keratoconus patients. Importantly, our results suggest that corneal thickness remained stable in paediatric keratoconus patients after ATE-CXL throughout the 3-year follow-up period.

In conclusion, this study demonstrates that ATE-CXL is a safe and effective treatment for the paediatric progressive keratoconus patients. Keratometry and corneal thickness remained stable during the 36-month follow-up period. However, we could not disregard $20 \%$ of paediatric eyes showed a tendency for progression at 3 years after ATECXL, which was a relatively big proportion comparing to those who were treated by C-CXL in some studies. The importance of CXL lies in the fact that it is a minimally invasive procedure. Compared with C-CXL, the ATE-CXL is more safe, non-invasive and makes patient more comfortable. We recommend careful follow-up to detect a decrease of the CXL-induced effect. In addition, it remains unclear how these progressive corneas after ATE-CXL would react to possible retreatment. One limitation to our study is the lack of a control group undergoing C-CXL. Further studies should evaluate the changes of in vivo corneal morphology and biomechanical properties following ATE-CXL treatment in paediatric keratoconus patients.

Contributors Study concept and design (MT and XZha); data collection (MT, WJ, XZho and LS); analysis and interpretation of data (MT, WJ and LS); writing the 
manuscript (MT and WJ); critical revision of the manuscript (XZha); statistical expertise (MT and WJ); administrative, technical or material support (XZha and XZho); supervision (XZho)

Funding This work was supported by XZho from the National Natural Science Foundation of China (grant no. 81570879); by XZha from the National Natural Science Foundation of China for Young Scholars (grant no. 11702063)

Competing interests None declared.

Patient consent for publication Parental/guardian consent obtained.

Ethics approval This study was approved by the Ethics Committee of the Eye and ENT Hospital of Fudan University and adhered to the tenets of the Declaration of Helsinki.

Provenance and peer review Not commissioned; externally peer reviewed.

Data availability statement Data are available in a public, open access repository. Data are available from the corresponding author (doctzhouxingtao@163.com) on reasonable request.

Open access This is an open access article distributed in accordance with the Creative Commons Attribution Non Commercial (CC BY-NC 4.0) license, which permits others to distribute, remix, adapt, build upon this work non-commercially, and license their derivative works on different terms, provided the original work is properly cited, appropriate credit is given, any changes made indicated, and the use is noncommercial. See: http://creativecommons.org/licenses/by-nc/4.0/.

\section{ORCID iD}

Xingtao Zhou http://orcid.org/0000-0002-3465-1579

\section{REFERENCES}

1 Rabinowitz YS. Keratoconus. Surv Ophthalmol 1998;42:297-319.

2 Tuft SJ, Moodaley LC, Gregory WM, et al. Prognostic factors for the progression of keratoconus. Ophthalmology 1994;101:439-47.

3 Reeves SW, Stinnett S, Adelman RA, et al. Risk factors for progression to penetrating keratoplasty in patients with keratoconus. Am J Ophthalmol 2005;140:607-11.

4 Léoni-Mesplié S, Mortemousque $B$, Touboul $D$, et al. Scalability and severity of keratoconus in children. Am J Ophthalmol 2012;154:56-62.

5 Wollensak G, Spoerl E, Seiler T. Riboflavin/ultraviolet-a-induced collagen crosslinking for the treatment of keratoconus. Am J Ophthalmol 2003:135:620-7.

6 Schuerch K, Tappeiner C, Frueh BE. Analysis of pseudoprogression after corneal cross-linking in children with progressive keratoconus. Acta Ophthalmol 2016;94: e592-9.

7 Wise S, Diaz C, Termote K, et al. Corneal cross-linking in pediatric patients with progressive keratoconus. Cornea 2016;35:1441-3.

8 Viswanathan D, Kumar NL, Males JJ. Outcome of corneal collagen crosslinking for progressive keratoconus in paediatric patients. Biomed Res Int 2014;2014:1-5.

9 Uçakhan Ömür Ö, Bayraktutar BN, Saglik A. Pediatric corneal collagen cross-linking: long-term follow-up of visual, refractive, and topographic outcomes. Cornea 2016;35:162-8.

10 Vinciguerra $\mathrm{P}$, Albé E, Frueh BE, et al. Two-year corneal cross-linking results in patients younger than 18 years with documented progressive keratoconus. Am J Ophthalmol 2012:154:520-6.

11 Henriquez MA, Rodríguez AM, Izquierdo L. Accelerated Epi-On versus standard Epi-Off corneal collagen cross-linking for progressive keratoconus in pediatric patients. Cornea 2017:36:1503-8.
12 Tian M, Jian W, Sun L, et al. One-year follow-up of accelerated transepithelial corneal collagen cross-linking for progressive pediatric keratoconus. BMC Ophthalmol $2018 ; 18: 75$

13 Gomes JAP, Tan D, Rapuano CJ, et al. Global consensus on keratoconus and ectatic diseases. Cornea 2015;34:359-69.

14 Zhang X, Sun L, Chen Y, et al. One-year Outcomes of Pachymetry and Epithelium Thicknesses after Accelerated (45 mW/cm(2)) Transepithelial Corneal Collagen Crosslinking for Keratoconus Patients. Sci Rep 2016;6:32692.

15 Sun L, Li M, Zhang X, et al. Transepithelial accelerated corneal collagen cross-linking with higher oxygen availability for keratoconus: 1-year results. Int Ophthalmol 2018:38:2509-17.

16 Shen $Y$, Jian W, Sun L, et al. One-year follow-up of changes in corneal densitometry after accelerated (45 $\mathrm{mW} / \mathrm{cm} 2)$ transepithelial corneal collagen cross-linking for keratoconus: a retrospective study. Cornea 2016;35:1434-40.

17 Al Suhaibani AH, Al-Rajhi AA, Al-Motowa S, et al. Inverse relationship between age and severity and sequelae of acute corneal hydrops associated with keratoconus. $\mathrm{Br}$ J Ophthalmol 2007;91:984-5.

18 Chatzis N, Hafezi F. Progression of keratoconus and efficacy of pediatric [corrected] corneal collagen cross-linking in children and adolescents. J Refract Surg 2012:28:753-8.

19 Ozgurhan EB, Kara N, Cankaya KI, et al. Accelerated corneal cross-linking in pediatric patients with keratoconus: 24-month outcomes. J Refract Surg 2014;30:843-9.

20 Kumar Kodavoor S, Arsiwala AZ, Ramamurthy D. One-year clinical study on efficacy of corneal cross-linking in Indian children with progressive keratoconus. Cornea 2014:33:919-22.

21 Koller T, Mrochen M, Seiler T. Complication and failure rates after corneal crosslinking. J Cataract Refract Surg 2009;35:1358-62.

22 Greenstein SA, Fry KL, Bhatt J, et al. Natural history of corneal haze after collagen crosslinking for keratoconus and corneal ectasia: scheimpflug and biomicroscopic analysis. J Cataract Refract Surg 2010;36:2105-14.

23 Zhao Y, Li M, Zhao J, et al. Posterior corneal elevation after small incision Lenticule extraction for moderate and high myopia. PLoS One 2016;11:e0148370.

24 Zhao Y, Jian W, Chen Y, et al. Three-year stability of posterior corneal elevation after small incision Lenticule extraction (SMILE) for moderate and high myopia. J Refract Surg 2017;33:84-8

25 Yu M, Chen M, Dai J. Comparison of the posterior corneal elevation and biomechanics after SMILE and LASEK for myopia: a short- and long-term observation. Graefes Arch Clin Exp Ophthalmol 2019;257:601-6.

26 Ozgurhan EB, Sezgin Akcay BI, Yildirim Y, et al. Evaluation of corneal stromal demarcation line after two different protocols of accelerated corneal collagen cross-linking procedures using anterior segment optical coherence tomography and confocal microscopy. J Ophthalmol 2014;2014:1-5.

27 Moramarco A, lovieno A, Sartori A, et al. Corneal stromal demarcation line after accelerated crosslinking using continuous and pulsed light. J Cataract Refract Surg 2015;41:2546-51.

28 Müller LJ, Pels E, Vrensen GF. The specific architecture of the anterior stroma accounts for maintenance of corneal curvature. Br J Ophthalmol 2001;85:437-43.

29 Antonios R, Fattah MA, Maalouf F, et al. Central corneal thickness after cross-linking using high-definition optical coherence tomography, ultrasound, and dual scheimpflug tomography: a comparative study over one year. Am J Ophthalmol 2016;167:38-47.

30 Rocha KM, Rocha KM, Perez-Straziota CE, Stulting RD, et al. Epithelial and stromal remodeling after corneal collagen cross-linking evaluated by spectral-domain OCT. J Refract Surg 2014;30:122-7. 